\title{
Design and Application of Variable Universe Fuzzy Controller Based on Cat Swarm Optimization
}

\author{
Haipeng Pan and Dongbin Jin \\ Zhejiang Sci-Tech University, Hangzhou 310018, China \\ Correspondence should be addressed to Dongbin Jin; alexjin6504@foxmail.com
}

Received 26 July 2015; Revised 2 February 2016; Accepted 16 February 2016

Academic Editor: Yan-Wu Wang

Copyright (C) 2016 H. Pan and D. Jin. This is an open access article distributed under the Creative Commons Attribution License, which permits unrestricted use, distribution, and reproduction in any medium, provided the original work is properly cited.

\begin{abstract}
A novel variable universe fuzzy controller based on cat swarm optimization (CSO-VUFC) is proposed to regulate the temperature of the reactor system, which is characterized by nonlinearity, large time delay, and uncertainty. In CSO-VUFC, firstly, corresponding contraction-expansion factors with the function form were, respectively, introduced for the input and output fuzzy universes of the controller. Then, cat swarm optimization was used to optimize the relevant parameter values in the contraction-expansion factor function to achieve the intelligence optimization of the contraction-expansion factors, based on the system performance test function as an evaluation index; the contradiction between the universe adjustment and control accuracy of the fuzzy controller will be effectively solved to achieve the online self-adjustment of the universe. The simulation results indicate that the variable universe adaptive fuzzy control method based on the cat swarm optimization has the features of high precision adjustment, short transient time, and hard real-time.
\end{abstract}

\section{Introduction}

A fuzzy controller, which is designed based on experts' experiences and is independent of the mathematical model of the controlled object, can be used to effectively control the objects with characteristics of nonlinearity, large time delay, and uncertainty. However, a fuzzy controller is essentially a differential controller and the contradiction between the number of control rules and accuracy leads to the deficiencies of low steady-state accuracy and poor adaptability [1].

In order to solve the above-mentioned problems, [2] presented a GA-based two-stage fuzzy temperature control algorithm for industrial furnaces, which can greatly reduce the number of fuzzy rules by taking advantages of hierarchical fuzzy control and taking full account of impact of many procedure parameters upon controlled variables. In [3], as to the difficulties of multiparameters and nonlinearities, a new fuzzy algorithm adopting two phase control strategies is proposed. One is the fuzzy feedforward control algorithm for decoupling control. The other is the expert control algorithm for the system, which obtain a good control performance. The paper [4] focuses on the development of a direct adaptive fuzzy-neural-network control (DAFNNC), and numerical simulations of a two-link robot manipulator actuated by DC servomotors are given to verify the effectiveness and robustness of the proposed methodology. In [5] $T$ describes an intelligent coordinated control (ICC) system, which is based on both fuzzy feedforward (fuzzy-FF) control and the fuzzy-PID feedback control, and the industrial application results show that this strategy achieves better performance in the specific range of load variations. The paper [6] presents guideline for designing IT2 fuzzy PI control system with the aforementioned structure that has zero steady-state error, which shows good performance.

In the past, many optimization algorithms in the computational intelligence area such as the genetic algorithm (GA), simulated annealing (SA), and particle swarm optimization (PSO) have been used to tune the control parameters in order to find an optimal performance [7]. Cat swarm optimization (CSO) algorithm was recently developed to show better for finding the global best solutions in comparison to some other evolutionary algorithms [8]. Cat swarm optimization (CSO) adopts a population-based stochastic search but has a different learning rule, with strong global and local searching ability. 
On the basis of the previous researchers, in this paper, a new variable universe fuzzy controller based on the cat swarm optimization (CSO-VUFC) is proposed and the temperature of a reactor is the controlled object, with the large time delay characteristics. Firstly, with analyzing and deciding the structure of contraction-expansion factor $f$, the contractionexpansion factor is introduced for changeable discourse. Then, cat swarm optimization was used to optimize relative parameters, based on the performance evaluation function of the system, so as to obtain a group of the relatively optimal fuzzy control parameters to make the tracing value reach the relatively ideal control effects. Finally, on the platform of the MATLAB software, taking temperature of the reactor as the controlled object, the simulation results indicate that the controller can promptly adapt the changes of the dynamic characteristics of the object and the control effect is good by comparing with the traditionally fuzzy control.

\section{Design Process of Variable Universe Fuzzy Controller Based on Cat Swarm Optimization}

2.1. Description of Variable Universe Fuzzy Control. Denote that $X_{i}=\left[-E_{i}, E_{i}\right]$ are the universes of input variables $x_{i}(i=$ $1,2,3, \ldots, n)$ and $Y=[-U, U]$ is the universe of output variable $y . A=A_{i j}(i=1,2,3, \ldots, n, j=1,2,3, \ldots, m)$ is the fuzzy partition on $X_{i}$, and $B=\left\{B_{i}\right\}$ is the fuzzy partition on $Y$. $A$ and $B$ are regarded as the linguistic variables so that a group of fuzzy inference rules is formed as follows [9]:

$$
\begin{aligned}
& \text { If } x_{1} \text { is } A_{1 j} \text {, and } x_{2} \text { is } A_{2 j}, \ldots \text {, and } x_{n} \text { is } A_{n j} \text {, } \\
& \text { then } y \text { is } B_{j}(j=1,2, \ldots, m) \text {. }
\end{aligned}
$$

By letting $X_{i j}$ be the peak point (the membership function value on this point is 1$)$ of $A_{i j}$ and $Y_{j}$ the peak point of $B_{j}(i=$ $1,2,3, \ldots, n, j=1,2,3, \ldots, m$ ), the fuzzy logic system (i.e., a fuzzy controller) based on (1) can be represented as an $n$-array piecewise interpolation function $F\left(x_{1}, x_{2}, \ldots, x_{n}\right)$ :

$$
\begin{aligned}
F\left(x_{1}, x_{2}, \ldots, x_{n}\right) & =y\left(x_{1}, x_{2}, \ldots, x_{n}\right) \\
& =\sum_{j=1}^{m} \prod_{i=1}^{n} A_{i j}\left(x_{i}\right) y_{i} .
\end{aligned}
$$

The so-called variable universe means that some universes, such as $x_{i}$ and $y$, can change along with changeable variables $x_{i}$ and $y[10]$, which is denoted by

$$
\begin{aligned}
X_{i}\left(x_{i}\right) & =\left[-\alpha_{i}\left(x_{i}\right) E_{i}, \alpha_{i}\left(x_{i}\right) E_{i}\right], \\
Y(y) & =[-\beta(y) U, \beta(y) U],
\end{aligned}
$$

where $\alpha_{i}\left(x_{i}\right)(i=1,2, \ldots, n)$ are input universe contraction factor and $\beta(y)$ is called the output universe contraction factor. $X_{i}, Y$ are original universes. The linguistic variables are varying together with the universe, as shown in Figure 1.

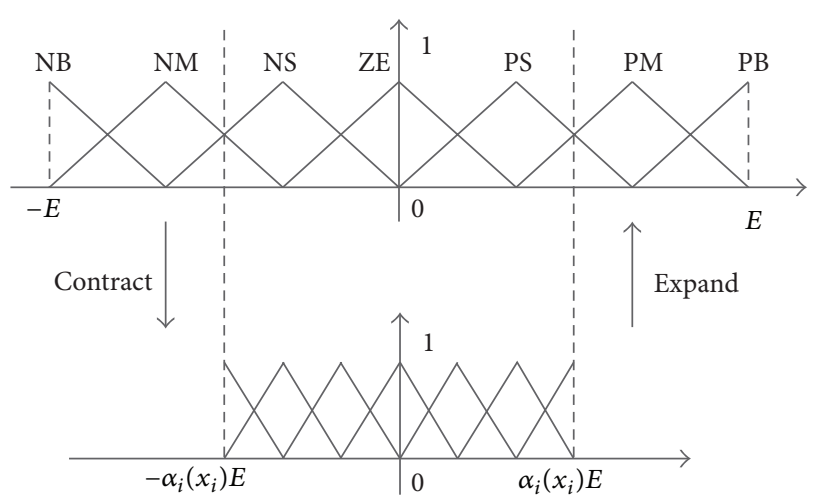

FIgURE 1: Illustration of variable universe.

Based on (1), (2), and Figure 1, a variable adaptive fuzzy controller can be represented as an $n$-array piecewise dynamic interpolation function in the following:

$$
y(x(t+1))=\beta(y(x(t+1))) \sum_{j=1}^{m} \prod_{i=1}^{n} A_{i j}\left[\frac{x_{i}(t)}{\alpha\left(x_{i}(t)\right)}\right],
$$

where $x(t)=x_{i}(t)(i=1,2,3, \ldots, n)$.

2.2. Choose Contraction-Expansion Factors. It is easy to find that $\alpha_{i}\left(x_{i}\right)$ and $\beta(y)$ affect the dynamic performance of a system. Thus how to determine the contraction-expansion factors becomes an important problem.

Generally speaking, a function $\alpha: X \rightarrow[0,1], x \rightarrow \alpha(x)$ is called a universe contraction-expansion factor on $X \in$ $[-E, E]$, if it satisfies the following conditions [11]:

Duality: $\alpha(x)=\alpha(-x)$.

Zero kept: $\alpha(x) \geq \xi>0, \xi>0$.

Monotonicity: $\alpha$ is strictly monotonically increasing on $[0, E]$.

$$
\begin{aligned}
& \text { Normality: } \alpha( \pm E)=1 . \\
& \text { Compatibility: }|x| \leq \alpha(x) E, \forall x \in X .
\end{aligned}
$$

At present, the contraction-expansion factors of universes have no unified form. The following equation is chosen as the contraction-expansion factor in this paper:

$$
\alpha_{1}(x)=\left(\frac{|x|}{E}\right)^{\tau}+\xi, \quad \tau>0, \xi>0 .
$$

It is easy to verify that (4) meets the axiomatic conditions to the arbitrary parameters, so it can be regarded as a universe contraction-expansion factor.

Generally, for a dual-input single-output fuzzy control system, the error $e$ and its change rate $e c$ are chosen to be the inputs and the output $y$ of the fuzzy controller is chosen to be the output.

Based on (2), we have

$$
\begin{aligned}
X_{1}(e) & =\left[-\alpha_{1}(e) E, \alpha_{1}(e) E\right], \\
X_{2}(e c) & =\left[-\alpha_{2}(e c) E C, \alpha_{2}(e) E C\right], \\
Y(e, e c) & =[-\beta(e, e c) U, \beta(e, e c) U] .
\end{aligned}
$$




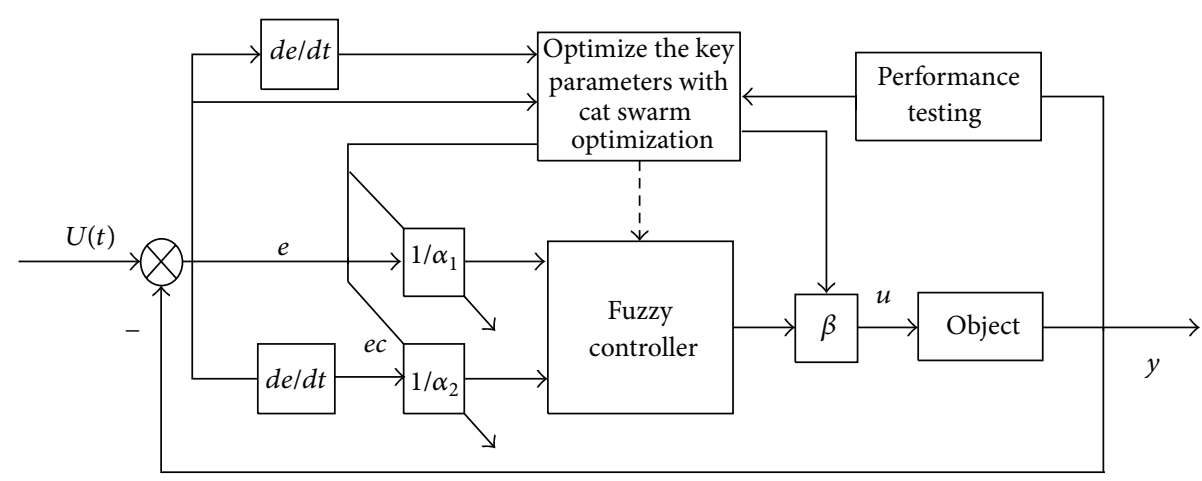

Figure 2: The structure of the CSO-VUFC.

Based on (4) and (5), the contraction-expression factors in this paper are obtained as follows:

$$
\begin{aligned}
\alpha_{1}(e) & =\left[\frac{|e|}{E}\right]^{k_{1}}, \\
\alpha_{2}(e) & =\left[\frac{|e c|}{E C}\right]^{k_{2}}, \\
\beta(e, e c) & =\left[\frac{|e|}{E}\right]^{k_{3}}\left[\frac{|e c|}{E C}\right]^{k_{4}},
\end{aligned}
$$

where $k_{1}, k_{2}, k_{3}, k_{4}$ are parameters to be optimized.

2.3. Cat Swarm Optimization. Cat swarm optimization (CSO) was proposed by Tsai et al. [12, 13], who got inspiration from observing the cat behaviors in daily life. In CSO, the cats' behaviors can be divided into two modes, tracing mode and seeking mode [14].

2.3.1. Seeking Mode. In the seeking mode, cats do not move, but just stay in a certain position and sense for the next best move, thus having only state and no velocity [15]. Some of the essential factors related to this mode are defined as follows:

seeking memory pool (SMP): the seeking memory size for each cat to consider the candidate coordinates,

seeking range of the selected dimension (SRD): the range to be varied for a selected dimension,

counts of dimension to change (CDC): the index of dimensions to be mutated.

The steps involved in the seeking mode are as follows.

Step 1 (copy cats' location). Generate $J$ copies of cats' location and then put them into SMP. The size of memory is $J$.

Step 2 (execute the mutation operator). Execute the mutation operator on the replicated copies based on SRD and CDC, which could make each copy reach a new location.

Step 3. Calculate the fitness values of all the copies in seeking memory pool.
Step 4 (execute the selection operator). Select the position relating to the best fitness value and move the cat to this location.

2.3.2. Tracing Mode. The tracing mode is a model established under the condition that cat is tracking a target, which uses a speed-displacement model to change the value in every dimension. $x_{k}^{(d)}(t)$ is a single cat's current position; $V_{i}=$ $\left\{v_{i}^{1}, v_{i}^{2}, v_{i}^{3}, \ldots, v_{i}^{L}\right\}$ is a single cat's current velocity; $x_{\text {best }}^{(d)}(t)$ is the "best" position of the whole cat population.

The steps involved in this mode are described as follows.

Step 1 (update the velocity). Every cat employs the following to update its velocity:

$$
\begin{array}{r}
v_{k}^{(d)}(t+1)=v_{k}^{(d)}(t)+c_{1} \times \operatorname{rand}\left(x_{\text {best }}^{(d)}(t)-x_{k}^{(d)}(t)\right), \\
d=1,2, \ldots, L,
\end{array}
$$

where $c_{1}$ is a constant, whose value is determined according to different problems; rand is the random number between 0 and 1 .

Step 2 (determine whether the velocity change is out of range). If so, it needs to be reset to a given boundary value.

Step 3 (update the location). According to the following, cat's location is updated:

$$
x_{k}^{d}(t+1)=x_{k}^{(d)}(t)+v_{k}^{(d)}(t+1) .
$$

2.4. The Structure of CSO-VUFC. The structure of CSOVUFC is illustrated in Figure 2. In Figure 2, cat optimization algorithm is used to optimize contraction-expansion factors $\alpha_{1}(e), \alpha_{2}(e c), \beta(e, e c)$. When universes of discourse change along with contraction-expansion factors, the precision of the fuzzy controller can be improved without changing the number of control rules.

$e$ is the error, $e c$ is the error rate, and $u$ is the output control parameter after defuzzification. $\alpha_{1} \alpha_{2}$ and $\beta$ are contractionexpansion factors. In this paper, the fuzzy controller is set as follows: 


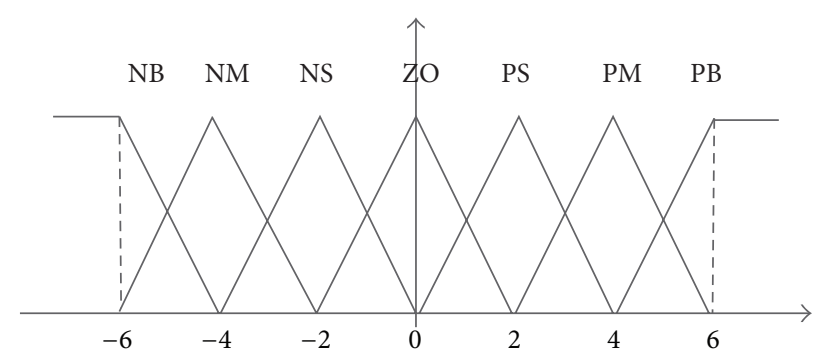

FIgURE 3: Fuzzy membership function.

(1) The universes of $E, E C$, and $U$ are made as $\{-6,-4,-2,0,+2,+4,+6\} ; \quad$ fuzzy quantizations are made as $\{\mathrm{NB}, \mathrm{NM}, \mathrm{NS}, \mathrm{ZO}, \mathrm{PS}, \mathrm{PM}, \mathrm{PB}\}$.

(2) The controller employed triangle membership function, as in Figure 3.

2.5. Controller Implementation of CSO-VUFC. The design idea of CSO-VUFC can be summarized as follows: firstly, determine the universes of error, error rate, and control variable; then, initialize the fuzzy queries; finally, apply the cat swarm optimization algorithm to optimize the parameters of contraction-expansion factors to make the system output close to the setting value. follows.

Implementation steps of CSO-VUFC are described as

Step 1. Separately identify the fuzzy universe of $E, E C$, and $U$ and initialize the related quantitative factors $K e, K e c$, and $\mathrm{Ku}$.

Step 2. Optimize $k_{1}, k_{2}, k_{3}, k_{4}$ with CSO, which consists of 4 steps:

(1) Initialize the parameters of CSO; the population size of the cat group is 100; dimension of cat is 4 and the mixture ratio of two modes is 0.3 ; the seeking range of the selected dimension is 0.2 ; and the memory pool size is 20 .

(2) Update the position and velocity of the cat group and calculate the fitness value of each cat; according to (7) and (8), choose the ITAE index [16] as the fitness function of parameter selection, as the following equation:

$$
\operatorname{ITAE}=\sum_{K=0}^{n}|e(k T)| \times k T \times T,
$$

where $T$ is sampling period, $k$ is sampling time, and $e(k T)$ is the error of the sampling time. The smaller the value of the ITAE is, the better the control effect of the fuzzy control will be.

(3) Separately calculate the fitness value of each cat $x_{k}^{(d)}(t)$ of two modes and search and record the current best position.

(4) Update the global optimal solution $x_{\text {best }}(t)$, after completing one search.
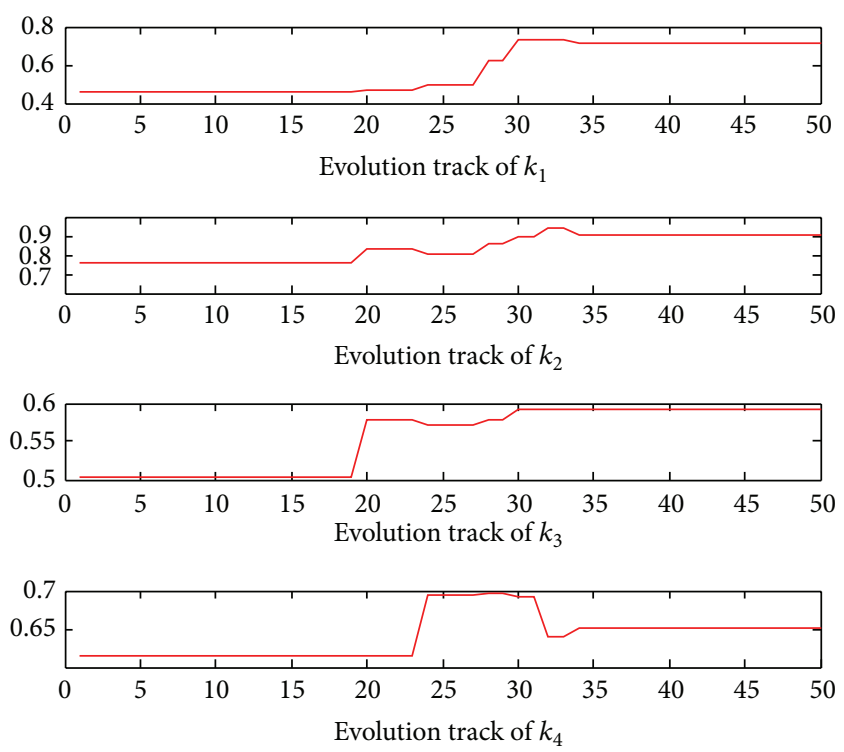

FIGURE 4: Evolution track of $k_{1}, k_{2}, k_{3}, k_{4}$.

Step 3. Determine if the current output is desired. If so, terminate the process and return the global optimal solutions; otherwise, return to Step 2.

\section{Simulation of the CSO-VUFC}

In this work, the experiments of optimizing relevant parameters of fuzzy controller by cat swarm optimization (CSO) were carried out based on MATLAB software. The temperature of the reactor, approximately a first-order lag process with dead time described in (10) is chosen as our control object:

$$
G(s)=\frac{K}{T s+1} e^{-\tau \cdot s},
$$

where $t$ parameter scaling factor $K=1.2$, time constant $T=$ 80 , and delay factor $\tau=120$.

3.1. The Analysis of Control Effect of CSO-VUFC. On the platform of the MATLAB software, from simulation of the CSO-VUFC controller, an optimal set of parameters is obtained and the evolution tracks of $k_{1}, k_{2}, k_{3}, k_{4}$ and ITAE are shown as in Figures 4 and 5, where

$$
\begin{aligned}
& k_{1}=0.71 ; \\
& k_{2}=0.90 ; \\
& k_{3}=0.59 ; \\
& k_{4}=0.65
\end{aligned}
$$

These optimized parameters are then applied in the design of the CSO-VUFC controller, and the controller is used to control the temperature of the reactor; the output is compared with the results of PID controller and traditional fuzzy controller (T-Fuzzy). 


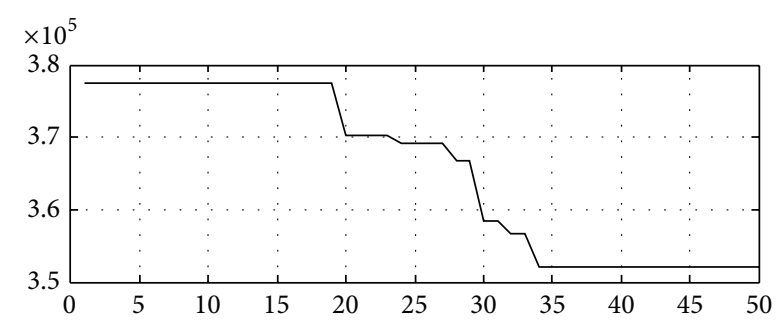

FIGURE 5: Evolution track of ITAE.

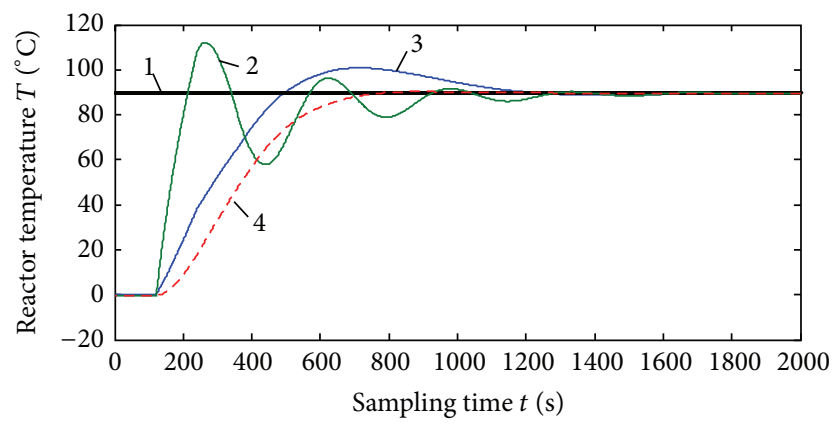
(1) The temperature system set
(3) T-Fuzzy
(2) PID
(4) CSO-VUFC

FIGURE 6: The curves of the temperature of three controllers.

TABLE 1: Compared results of three controllers.

\begin{tabular}{lccc}
\hline & Rise time $(\mathrm{S})$ & Overshoot $\left({ }^{\circ} \mathrm{C}\right)$ & Regulation time $(\mathrm{S})$ \\
\hline PID & 56 & 16 & 1323 \\
T-Fuzzy & 214 & 9 & 1146 \\
CSO-VUFC & 278 & 0.2 & 784 \\
\hline
\end{tabular}

When the system is set with a step signal at $90^{\circ} \mathrm{C}$, the simulation results of three methods are shown in Figure 6. The overshoot and regulation time of three fuzzy controllers are given in Table 1.

From Figure 6 and Table 1, the system under the PID controller has a big overshoot of $12.6^{\circ} \mathrm{C}$. The traditional fuzzy controller (T-Fuzzy) method decreases the overshoot, but the regulation time is also long. The variable universe fuzzy controller based on the cat swarm optimization (CSOVUFC) not only has a smaller overshoot but also improves the regulation time. The regulation time of the system shortens to 784 seconds, and the overshoot of the system is less than $1^{\circ} \mathrm{C}$. So, variable universe fuzzy controller based on cat swarm optimization (CSO-VUFC) shows better temperature following and steady-state performance.

\section{Conclusion}

In this paper, as to the questions that the fuzzy universe for the conventional fuzzy controller cannot be adjusted in time at the time of the system operation, the variable universe fuzzy control method based on the cat swarm optimization is proposed. By, respectively, introducing the corresponding contraction-expansion factors with the function form in the input and output fuzzy universes of the controller. Then, cat swarm optimization was used to optimize the relevant parameter values in the contraction-expansion factor function to achieve the intelligence optimization of the contraction-expansion factors, based on the system performance test function as an evaluation index; the contradiction between the universe adjustment and control accuracy of the fuzzy controller will be effectively solved to achieve the online self-adjustment of the universe. The simulation results indicate that the variable universe adaptive fuzzy control method based on the cat swarm optimization has the features of high precision adjustment, short transient time, and hard real-time.

\section{Competing Interests}

The authors declare that they have no competing interests.

\section{Acknowledgments}

This paper is supported by Zhejiang Provincial Natural Science Foundation of China (Grant no. LZ15F030005). Thanks are due to Dr. Keyu Pan, for his contribution to the paper by text sorting, proofreading, and modifying icons.

\section{References}

[1] H.-G. Guo and B.-J. Zhang, "Observer-based variable universe adaptive fuzzy controller without additional dynamic order," International Journal of Automation and Computing, vol. 11, no. 4, pp. 418-425, 2014.

[2] X. Peng, Z. Mo, and L. Xiao, "Research and application on GA-based two-stage fuzzy temperature control system for a type of industrial furnace," in Proceedings of the International Conference on Electrical and Control Engineering (ICECE '10), pp. 1558-1561, IEEE, Wuhan, China, June 2010.

[3] H. Zhou, W. Chen, and K. Fang, "A fuzzy control algorithm for collecting main pressure controlling using expert control," in Proceedings of the International Conference on Intelligent Computation Technology and Automation (ICICTA '10), vol. 1, pp. 104-107, Changsha, China, May 2010.

[4] R.-J. Wai, Z.-W. Yang, and C.-Y. Shih, "Direct adaptive fuzzyneural-network control for robot manipulator by using only position measurements," in Proceedings of the 5th IEEE Conference on Industrial Electronics and Applications (ICIEA '10), pp. 689-694, IEEE, Taichung, Taiwan, June 2010.

[5] X.-F. Li, S.-H. Chen, and Q. Zhong, "The coordinated control of circulating fluidized bed boiler with fuzzy feedforward control," in Proceedings of the Annual Meeting of the North American Fuzzy Information Processing Society (NAFIPS '11), pp. 1-8, IEEE, El Paso, Tex, USA, March 2011.

[6] C. M. Yip and W. W. Tan, "Ensuring zero steady state error in interval type-2 fuzzy PI control system with non-symmetrical fuzzy sets," in Proceedings of the IEEE International Conference on Fuzzy Systems (FUZZ-IEEE '15), pp. 1-8, IEEE, Istanbul, Turkey, August 2015.

[7] M. Dorigo, M. Birattari, C. Blum, M. Clerc, Th. Stützle, and A. Winfield, Eds., Ant Colony Optimization and Swarm Intelligence: 6th International Conference, ANTS 2008, Brussels, Belgium, September 22-24, 2008, Proceedings, Springer, 2008. 
[8] Z.-L. Gaing, "Particle swarm optimization to solving the economic dispatch considering the generator constraints," IEEE Transactions on Power Systems, vol. 18, no. 3, pp. 1187-1195, 2003.

[9] H.-X. Li and C. L. P. Chen, "The equivalence between fuzzy logic systems and feedforward neural networks," IEEE Transactions on Neural Networks, vol. 11, no. 2, pp. 356-365, 2000.

[10] B. Hou and Y. Gao, "Variable universe fuzzy controller with correction factors for ball and beam system," in Proceedings of the 3rd International Workshop on Intelligent Systems and Applications (ISA '11), pp. 1-4, IEEE, Wuhan, China, May 2011.

[11] H. Li, X.-D. Su, and H.-T. Jiang, "Asymmetric variable universe adaptive landing fuzzy controller for carrier-based aircraft," International Journal of Smart Home, vol. 9, no. 5, pp. 31-40, 2015.

[12] P.-W. Tsai and V. Istanda, "Review on cat swarm optimization algorithms," in Proceedings of the 3rd International Conference on Consumer Electronics, Communications and Networks (CECNet '13), pp. 564-567, IEEE, Xianning, China, November 2013.

[13] P. M. Pradhan and G. Panda, "Solving multiobjective problems using cat swarm optimization," Expert Systems with Applications, vol. 39, no. 3, pp. 2956-2964, 2012.

[14] S. K. Saha, S. P. Ghoshal, R. Kar, and D. Mandal, "Cat swarm optimization algorithm for optimal linear phase FIR filter design," ISA Transactions, vol. 52, no. 6, pp. 781-794, 2013.

[15] S. Bilgaiyan, S. Sagnika, and M. Das, "Workflow scheduling in cloud computing environment using Cat Swarm Optimization," in Proceedings of the 4th IEEE International Advance Computing Conference (IACC '14), pp. 680-685, IEEE, Gurgaon, India, February 2014.

[16] B. K. Sahu, S. Pati, and S. Panda, "Hybrid differential evolution particle swarm optimisation optimised fuzzy proportionalintegral derivative controller for automatic generation control of interconnected power system," IET Generation, Transmission and Distribution, vol. 8, no. 11, pp. 1789-1800, 2014. 


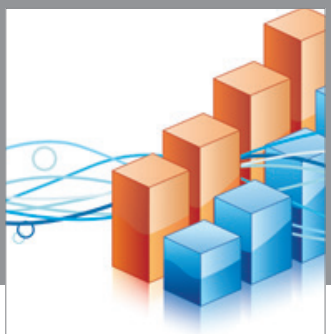

Advances in

Operations Research

vatem alat4

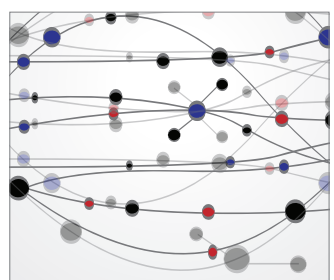

\section{The Scientific} World Journal
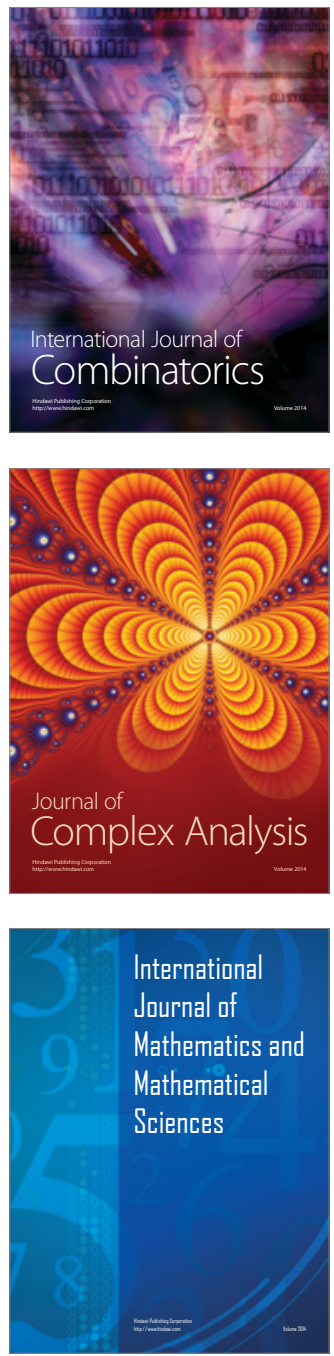
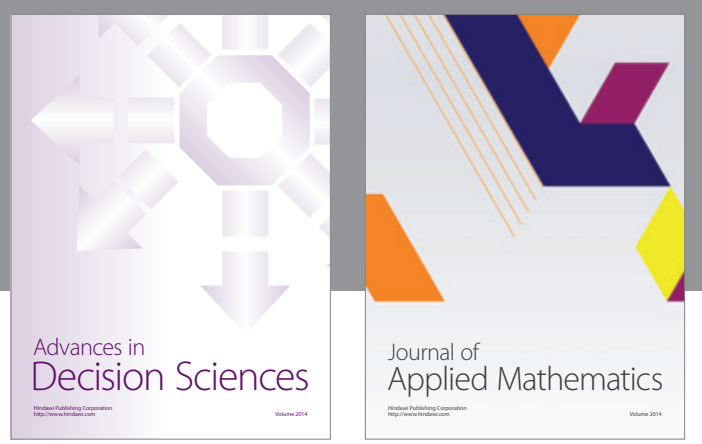

Algebra

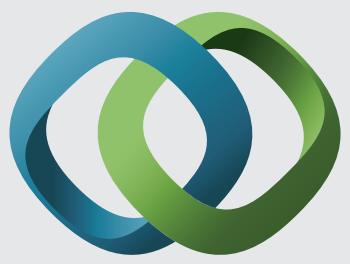

\section{Hindawi}

Submit your manuscripts at

http://www.hindawi.com
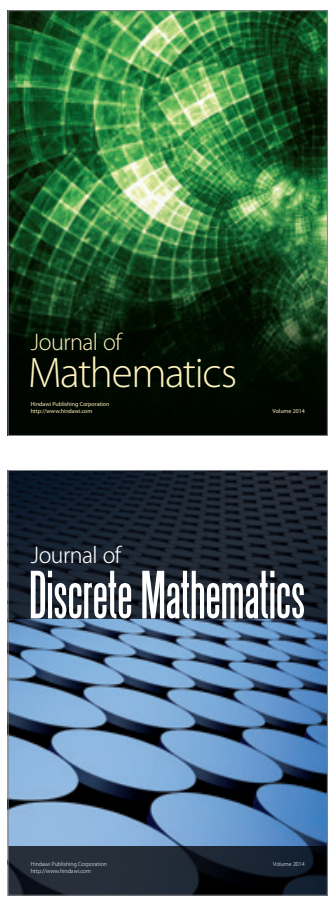

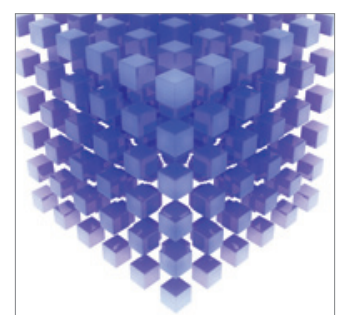

Mathematical Problems in Engineering
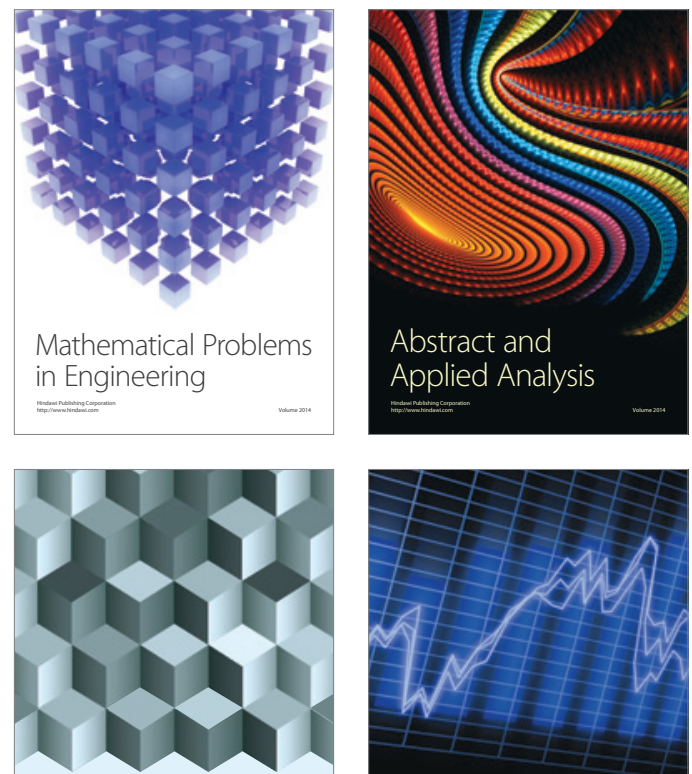

Journal of

Function Spaces

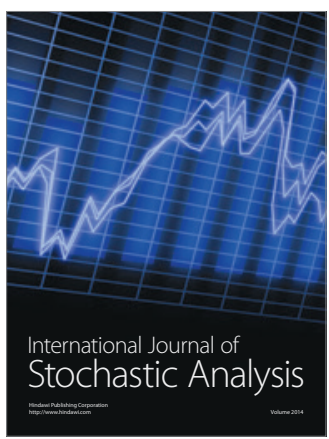

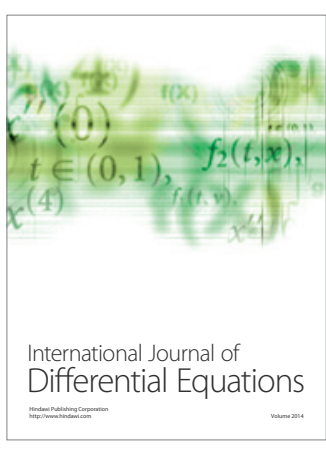
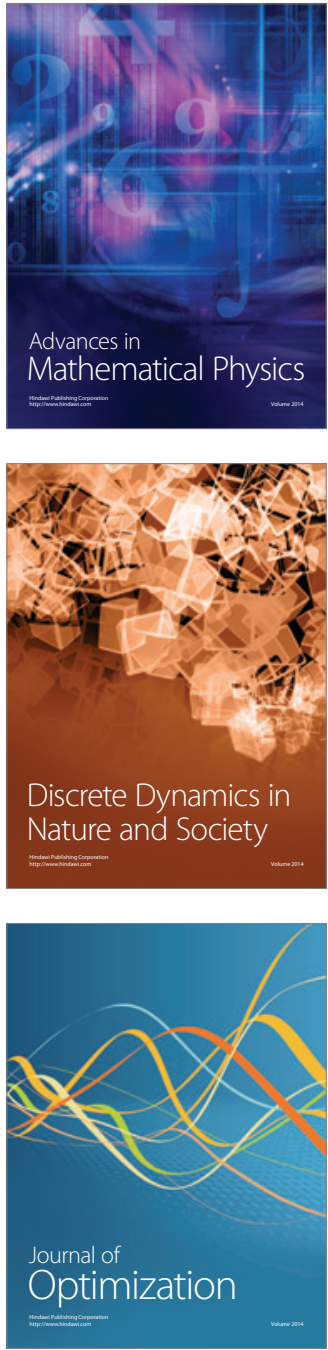